\title{
Likelihood analysis of cosmic shear on simulated and VIRMOS-DESCART data ${ }^{\star}$
}

\author{
L. Van Waerbeke ${ }^{1,2}$, Y. Mellier ${ }^{1,3}$, R. Pelló ${ }^{4}$, U.-L. Pen ${ }^{2}$, H. J. McCracken ${ }^{5,6,7}$, and B. Jain ${ }^{8}$ \\ 1 Institut d'Astrophysique de Paris, 98 bis boulevard Arago, 75014 Paris, France \\ 2 Canadian Institute for Theoretical Astrophysics, 60 St George Str., Toronto, M5S 3H8, Canada \\ 3 Observatoire de Paris, LERMA, 61 avenue de l'Observatoire, 75014 Paris, France \\ ${ }^{4}$ Observatoire Midi-Pyrénées, UMR 5572, 14 avenue E. Belin, 31400 Toulouse, France \\ 5 Department of Astronomy, University of Bologna, via Ranzani 1, 40127 Bologna, Italy \\ ${ }^{6}$ Observatorio Astronomico di Bologna, via Ranzani 1, 40127 Bologna, Italy \\ 7 Laboratoire d'Astrophysique de Marseille, Traverse du Siphon, 13376 Marseille Cedex 12, France \\ 8 Department of Physics and Astronomy, University of Pennsylvania, 209 S. 33rd Street, Philadelphia, PA 19104, USA
}

Received 28 February 2002 / Accepted 4 June 2002

\begin{abstract}
We present a maximum likelihood analysis of cosmological parameters from measurements of the aperture mass up to 35 arcmin using simulated and real cosmic shear data. A four-dimensional parameter space is explored which examines the mean density $\Omega_{\mathrm{M}}$, the mass power spectrum normalisation $\sigma_{8}$, the shape parameter $\Gamma$ and the redshift of the sources $z_{s}$. Constraints on $\Omega_{\mathrm{M}}$ and $\sigma_{8}\left(\right.$ resp. $\Gamma$ and $z_{\mathrm{s}}$ ) are provided by marginalising over $\Gamma$ and $z_{\mathrm{s}}\left(\right.$ resp. $\Omega_{\mathrm{M}}$ and $\sigma_{8}$ ). For a flat $\Lambda$ CDM cosmologies, using a photometric redshift prior for the sources and $\Gamma \in[0.1,0.4]$, we find $\sigma_{8}=(0.57 \pm 0.04) \Omega_{\mathrm{M}}^{(0.24 \mp 0.18) \Omega_{\mathrm{M}}-0.49}$ at the $68 \%$ confidence level (the error budget includes statistical noise, full cosmic variance and residual systematics). The estimate of $\Gamma$, marginalised over $\Omega_{\mathrm{M}} \in[0.1,0.4], \sigma_{8} \in[0.7,1.3]$ and $z_{\mathrm{s}}$ constrained by photometric redshifts, gives $\Gamma=0.25 \pm 0.13$ at $68 \%$ confidence. Adopting $h=0.7$, a flat universe, $\Gamma=0.2$ and $\Omega_{\mathrm{m}}=0.3$ we find $\sigma_{8}=0.98 \pm 0.06$. Combined with CMB measurements, our results suggest a non-zero cosmological constant and provide tight constraints on $\Omega_{\mathrm{M}}$ and $\sigma_{8}$. Finally, we compare our results to the cluster abundance ones, and discuss the possible discrepancy with the latest determinations of the cluster method. In particular we point out the actual limitations of the mass power spectrum prediction in the non-linear regime, and the importance in improving this.
\end{abstract}

Key words. cosmology: dark matter - cosmology: gravitational lensing

\section{Introduction}

In the standard cosmological picture, the structures in the Universe grow from the gravitational collapse of initial Gaussian density perturbations. The properties of the distribution of mass at low redshifts are expected to express the latest and one of the most explicit footprints of the structure formation process; it is for this reason that observations from cosmological surveys can be challenged against theoretical predictions resulting from this paradigm.

For example, a direct observation of the mass distribution in structures is believed to be an unequivocal test of the cosmological scenario of structure formation. If this picture is correct, weak gravitational lensing produced on distant

Send offprint requests to: L. Van Waerbeke,

e-mail: waerbeke@iap.fr

* Based on observations obtained at the Canada-France-Hawaii Telescope (CFHT), which is operated by the National Research Council of Canada (NRCC), the Institut des Sciences de l'Univers (INSU) of the Centre National de la Recherche Scientifique (CNRS) and the University of Hawaii (UH), and at the European Southern Observatory telescopes Very Large Telescope (VLT) and the New Technology Telescope (NTT). galaxies by large scale structures is a direct probe of dark matter, regardless of the light distribution. It is therefore a robust technique to challenge current cosmological models. In particular, it can reliably probe small angular scales and can be used to investigate the transition to the quasi-linear and non-linear regimes, where comparison between observations and cosmological models is still challenging.

The cosmological origin of the coherent distortion fields detected in cosmic shear surveys is now firmly established (Bacon et al. 2000; Haemmerle et al. 2002; Hoekstra et al. 2002; Kaiser et al. 2000; Maoli et al. 2001; Pen et al. 2002; Rhodes et al. 2001; Van Waerbeke et al. 2001, 2001b; Wittman et al. 2000). Van Waerbeke et al. (2001b) have shown that the measurements provided by different statistical estimators of the distortion signal are consistent with the gravitational lensing hypothesis with a high confidence level, and present-day datasets can already constrain cosmological parameters. Their joint estimate of the mass density $\Omega_{\mathrm{M}}$ and the power spectrum normalisation $\sigma_{8}$ led to consistent results with the cluster abundance constraints (Pierpaoli et al. 2001) and confirmed earlier attempts by Maoli et al. (2001) and Rhodes et al. (2001) using ESO-VLT/CFHT and HST data respectively. A recent 
measurement performed on a shallow survey (therefore very different in depth) confirmed this agreement (Hoekstra et al. 2001, 2002).

Until now, cosmological parameter estimation from cosmic shear relied on prior knowledge of the slope of the mass power spectrum $\Gamma$ and/or the mean redshift $\bar{z}_{\mathrm{s}}$ of the lensed galaxy population. In fact, the statistical properties of cosmic shear depend significantly on these quantities (Kaiser 1992; Bernardeau et al. 1997; Jain \& Seljak 1997), so any prior on these parameters may have a serious impact on the cosmological parameter estimation. For instance changing the shape of the power spectrum in either direction would favour low or high matter densities by changing the normalisation accordingly. This ambiguity expresses a degeneracy between the normalisation and the mass density, which depends on the choice of $\Gamma$ (Van Waerbeke et al. 2001b). Jain \& Seljak (1997) addressed this issue by pointing out that a measurement of the cosmic shear in both linear and non-linear scales could break the degeneracy, so that one in principle recovers simultaneously $\Gamma, \sigma_{8}$ and $\Omega_{\mathrm{M}}$ from the shear variance alone. Unfortunately, the redshift of the sources is also a strongly degenerate parameter with $\sigma_{8}$, which prevents shear variance analysis from providing an unequivocal discrimination of cosmological models. Stringent constraints on the cosmological parameters from the shear variance are possible only with an accurate knowledge of the source redshifts and a measurement which extends over a large range of scales.

In this paper we carry out a full maximum likelihood analysis of cosmic shear data over the four parameters $\Omega_{\mathrm{M}}, \sigma_{8}, \Gamma$, gand $\bar{z}_{\mathrm{s}}$ for flat and open cosmologies. Using both simulations and observations, we study slices and projections in this parameter space and discuss the reliability of cosmological constraints derived from catalogues having similar size and depth to current cosmic shear surveys. In particular we provide an estimate of $\Omega_{\mathrm{M}}$ and $\sigma_{8}$ by marginalising over the power spectrum shape and source redshifts. The improvement of our knowledge of the source redshifts is crucial in achieving better accuracy for the other parameters. In this work we use photometric redshifts ${ }^{1}$ to set priors on the source redshift distribution.

The paper is organised as follows. Section 2 presents a brief summary of some theoretical concepts and introduces the shear estimators used throughout the paper. Section 3 describes the data and how shear quantities were obtained from the survey catalogue. The likelihood method and the details of the priors are presented in Sect. 4. Section 5 shows and discusses the results on the parameter estimates on both simulated and real surveys. Finally, our conclusions are outlined in Sect. 6.

\section{Theory}

Following the notation in Schneider et al. (1998), we define the power spectrum of the convergence as

$$
\begin{aligned}
P_{K}(k)= & \frac{9}{4} \Omega_{0}^{2} \int_{0}^{w_{\mathrm{H}}} \frac{\mathrm{d} w}{a^{2}(w)} P_{3 \mathrm{D}}\left(\frac{k}{f_{\mathrm{K}}(w)} ; w\right) \\
& \times\left[\int_{w}^{w_{\mathrm{H}}} \mathrm{d} w^{\prime} n\left(w^{\prime}\right) \frac{f_{\mathrm{K}}\left(w^{\prime}-w\right)}{f_{\mathrm{K}}\left(w^{\prime}\right)}\right]^{2},
\end{aligned}
$$

\footnotetext{
1 Derived from other data sets.
}

where $f_{\mathrm{K}}(w)$ is the comoving angular diameter distance out to a distance $w\left(w_{\mathrm{H}}\right.$ is the horizon distance), and $n(w(z))$ is the redshift distribution of the sources given in Eq. (13). $P_{3 \mathrm{D}}(k)$ is the non-linear mass power spectrum computed according to Peacock \& Dodds (1996), and $k$ is the 2-dimensional wave vector perpendicular to the line-of-sight. The top-hat shear variance (computed using a smoothing window of radius $\theta_{\mathrm{c}}$ ) and the shear correlation function can be written as

$$
\begin{aligned}
& \left\langle\gamma^{2}\right\rangle=\frac{2}{\pi \theta_{\mathrm{c}}^{2}} \int_{0}^{\infty} \frac{\mathrm{d} k}{k} P_{\kappa}(k)\left[J_{1}\left(k \theta_{\mathrm{c}}\right)\right]^{2}, \\
& \langle\gamma \gamma\rangle_{\theta}=\frac{1}{2 \pi} \int_{0}^{\infty} \mathrm{d} k k P_{\kappa}(k) J_{0}(k \theta) .
\end{aligned}
$$

Because the weak distortion field can be generated by nonlensing mechanisms, it is important to measure separately the $E$ and $B$ components of the shear. These modes were introduced initially to test for the gravitational origin of the lensing signal (Crittenden et al. 2002) since a potential gravitational field is expected to produce only curl-free shear patterns ( $E$ mode). Any measurable $B$ mode can be interpreted as a measurement of residual systematics in the data (Point Spread Function correction, intrinsic alignment or anything else) must be removed from the weak lensing signal prior to cosmological interpretation of cosmic shear data.

The extraction of both modes is not trivial. The $E$ and $B$ mode decompositions of the top-hat shear variance, and of the shear correlation function given in Eqs. (2), (3) are only defined up to a integration constant (see Crittenden et al. 2002; Pen et al. 2002). This constant depends on the extrapolated cosmic shear signal either at small $(<30 \operatorname{arcsec})$ or large ( $>1$ degree) scales. These boundary conditions turn out to be a severe limitation that hampers reliable derivations of both modes from our present-day data because we do not cover very large angular scales and we still suffer from systematics on very small scales that are not well understood. As pointed out by Pen et al. (2002), the only unambiguous $E$ and $B$ mode decomposition can be carried out by using the aperture mass statistic, $M_{\mathrm{ap}}$ :

$M_{\text {ap }}=\int_{\theta<\theta_{\mathrm{c}}} \mathrm{d}^{2} \boldsymbol{\theta} \kappa(\boldsymbol{\theta}) U(\theta)$,

where $\kappa(\boldsymbol{\theta})$ is the convergence field, and $U(\theta)$ is the zero mass aperture window (Schneider et al. 1998):

$U(\theta)=\frac{9}{\pi \theta_{\mathrm{c}}^{2}}\left(1-\frac{\theta^{2}}{\theta_{\mathrm{c}}^{2}}\right)\left(\frac{1}{3}-\frac{\theta^{2}}{\theta_{\mathrm{c}}^{2}}\right)$.

This estimator was introduced in Kaiser et al. (1994) to study clusters of galaxies, but is it also useful for cosmic shear analysis (Schneider et al. 1998).

$\left\langle M_{\mathrm{ap}}^{2}\right\rangle$ can be calculated directly from the shear $\gamma$ without the need for a mass reconstruction. It can be rewritten as a function of the shear if we express $\gamma=\left(\gamma_{\mathrm{t}}, \gamma_{\mathrm{r}}\right)$ in the local frame of the line connecting the aperture center to the galaxy. $M_{\text {ap }}$ can therefore be expressed as function of $\gamma_{\mathrm{t}}$ only (Miralda-Escude 1991; Kaiser 1992):

$M_{\text {ap }}=\int_{\theta<\theta_{\mathrm{c}}} \mathrm{d}^{2} \boldsymbol{\theta} \gamma_{\mathrm{t}}(\boldsymbol{\theta}) Q(\theta)$, 
where the filter $Q(\theta)$ is given from $U(\theta)$ :

$Q(\theta)=\frac{2}{\theta^{2}} \int_{0}^{\theta} \mathrm{d} \theta^{\prime} \theta^{\prime} U\left(\theta^{\prime}\right)-U(\theta)$.

The aperture mass variance is related to the convergence power spectrum (Eq. (1)) by:

$\left\langle M_{\mathrm{ap}}^{2}\right\rangle=\frac{288}{\pi \theta_{\mathrm{c}}^{4}} \int_{0}^{\infty} \frac{\mathrm{d} k}{k^{3}} P_{\kappa}(k)\left[J_{4}\left(k \theta_{\mathrm{c}}\right)\right]^{2}$.

The $B$-mode is obtained by replacing $\gamma_{\mathrm{t}}$ with $\gamma_{\mathrm{r}}$ in Eq. (6). Although this estimator is robust and does not depend on an unknown integration constant, it is less sensitive to cosmological parameters than the top-hat variance or the shear correlation functions (Van Waerbeke et al. 2001b).

\section{Measurements}

We use the observations carried out within the VIRMOSDESCART project ${ }^{2}$ by the VIRMOS ${ }^{3}$ imaging and spectroscopic survey. The data cover an effective area of $8.5 \mathrm{sq} \mathrm{deg}$ in the $I$-band, with a limiting magnitude $I_{\mathrm{AB}}=24.5$. Technical details of the data set are given in Van Waerbeke et al. (2001b). We applied a bright magnitude cut at $I_{\mathrm{AB}}=21$ in order to exclude the foreground objects from the source galaxies. Galaxy shapes are measured and analysed (using the method described in Kaiser et al. 1995), to which we refer for technical details.

The location of the $i$ th galaxy is given by $\boldsymbol{\theta}_{i}$, the ellipticity by $\mathbf{e}\left(\boldsymbol{\theta}_{i}\right)=\left(e_{1}, e_{2}\right)$, and its weight $w_{i}$. The ellipticity is an unbiased estimate of the shear $\boldsymbol{\gamma}\left(\boldsymbol{\theta}_{i}\right)$. The quantity measured from the data are the binned tangential and radial shear correlation functions. They are given by a sum over galaxy pairs $\left(\boldsymbol{\theta}_{i}, \boldsymbol{\theta}_{j}\right)$

$\xi_{\mathrm{tt}}(r)=\frac{\sum_{i, j} w_{i} w_{j} e_{\mathrm{t}}\left(\boldsymbol{\theta}_{i}\right) \cdot e_{\mathrm{t}}\left(\boldsymbol{\theta}_{j}\right)}{\sum_{i, j} w_{i} w_{j}}$
$\xi_{\mathrm{rr}}(r)=\frac{\sum_{i, j} w_{i} w_{j} e_{\mathrm{r}}\left(\boldsymbol{\theta}_{i}\right) \cdot e_{\mathrm{r}}\left(\boldsymbol{\theta}_{j}\right)}{\sum_{i, j} w_{i} w_{j}}$,

where $r=\left|\boldsymbol{\theta}_{i}-\boldsymbol{\theta}_{j}\right|$, and $\left(e_{\mathrm{t}}, e_{\mathrm{r}}\right)$ are the tangential and radial ellipticities defined in the frame of the line connecting a pair of galaxies. The weights $w_{i}$ are computed for each galaxy from the intrinsic ellipticity variance $\sigma_{\mathrm{e}}^{2}$ and the rms of the ellipticity PSF correction $\sigma_{\epsilon}^{2}$. We have $\sigma_{\mathrm{e}} \simeq 0.4$ from the data, and we define the weights as:

$w_{i}=\frac{1}{\sigma_{\mathrm{e}}^{2}+\sigma_{\epsilon^{2}}}$.

To compute $\sigma_{\epsilon}$ for each galaxy we divide the galaxy sizemagnitude parameter space into cells of constant object number (typically 30 galaxies per cell). For each cell we then compute the rms of the ellipticity correction among the galaxies

\footnotetext{
2 http://terapix.iap.fr/DESCART

3 http://www .astrsp-mrs.fr
}
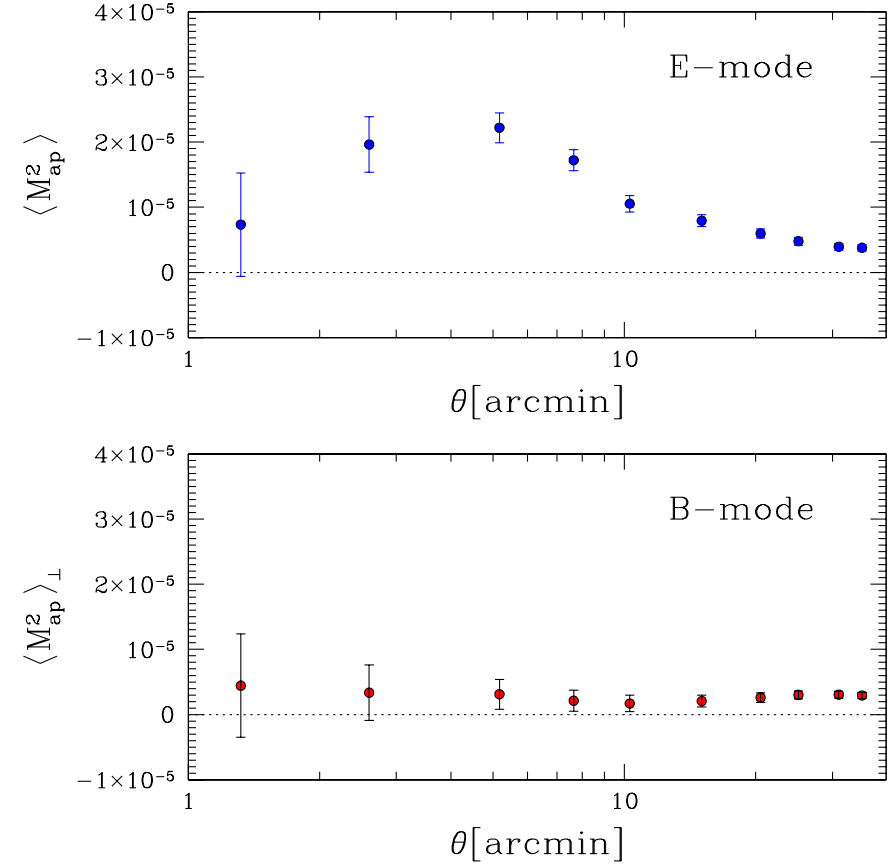

Fig. 1. The uppper panel shows the aperture mass statistic $\left\langle M_{\mathrm{ap}}^{2}\right\rangle(E-$ mode) and the lower the aperture mass $\left\langle M_{\mathrm{ap}}^{2}\right\rangle_{\perp}$ computed with galaxies rotated by 45 degrees ( $B$-mode). Error bars are $1-\sigma$ statistical errors.

in the cell. This choice of parameter space is motivated by the fact that the isotropic PSF correction (the so-called $P_{\gamma}$ term) is mainly sensitive to the size and magnitude of the galaxies (see Van Waerbeke et al. 2001b).

To compute the aperture mass from the data using Eq. (9), we define $\xi_{+}(r)$ and $\xi_{-}(r)$ which are respectively the sum and the difference of the two correlation functions:

$\xi_{+}(r)=\xi_{\mathrm{tt}}(r)+\xi_{\mathrm{rr}}(r) ; \quad \xi_{-}(r)=\xi_{\mathrm{tt}}(r)-\xi_{\mathrm{rr}}(r)$.

Both $\xi_{+}(r)$ and $\xi_{-}(r)$ are computed from a summation of the correlation function defined in Eq. (11), while the $E$ and $B$ modes aperture mass are derived by integration of the correlation functions with an appropriate window (see Crittenden et al. 2002 for general derivations and Pen et al. 2002 for a practical application to our filter).

The $E$ mode aperture mass is

$\left\langle M_{\mathrm{ap}}^{2}\right\rangle=\pi \int_{0}^{2 \theta_{\mathrm{c}}} r \mathrm{~d} r \mathcal{W}(r) \xi_{+}(r)+\pi \int_{0}^{2 \theta_{\mathrm{c}}} r \mathrm{~d} r \tilde{\mathcal{W}}(r) \xi_{-}(r)$,

where $\mathcal{W}(r)$ and $\tilde{\mathcal{W}}(r)$ are given in Crittenden et al. $(2002)^{4}$. The $B$-mode is obtained by changing the sign of the second term in Eq. (12) (which is equivalent to the 45 degrees rotation test, or else $\gamma_{\mathrm{t}} \rightarrow \gamma_{\mathrm{r}}$ ).

Figure 1 shows the $E$ mode (top) and $B$ mode (bottom) measured in our galaxy sample. Using the $B$-mode measurement, we found the source of the residual systematics at $3-4^{\prime}$ reported by Van Waerbeke et al. (2001b) and Pen et al. (2002): it was caused by the third order polynomial fit to the PSF, which

\footnotetext{
4 Useful expressions using similar formalism as this work can be found in Schneider et al. (2002).
} 

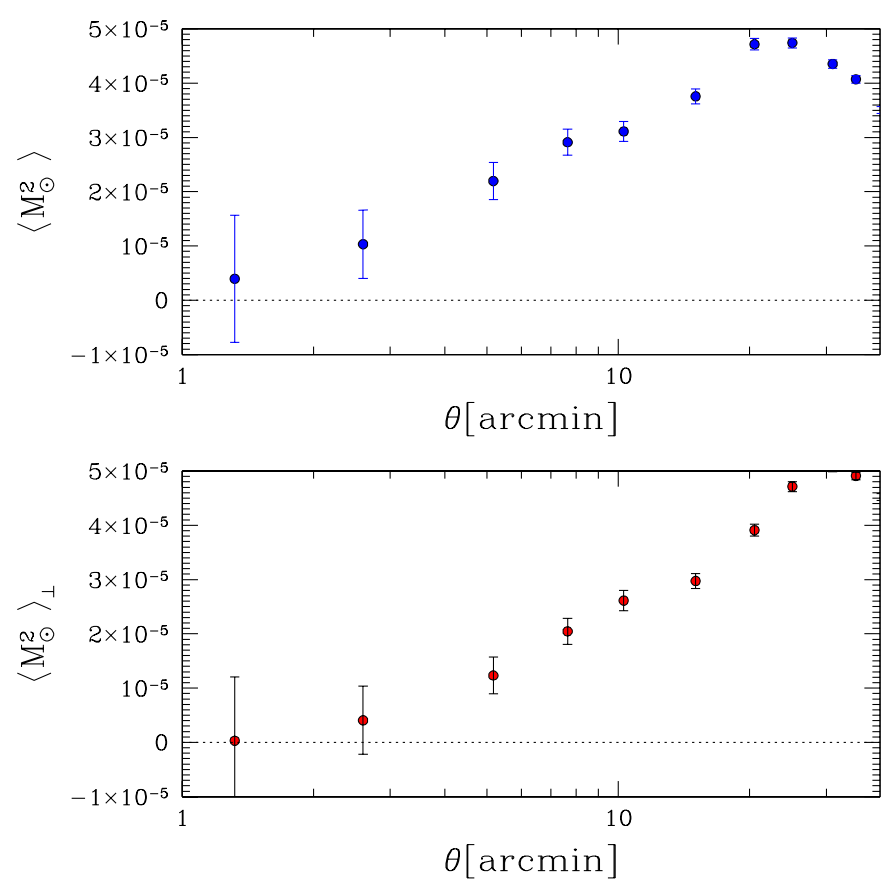

Fig. 2. Aperture mass statistic applied to stars before PSF correction. This plot can be compared to Fig. 1, which shows the same mode analysis on galaxies corrected from the PSF anisotropy. Note that the $\mathrm{E}$ and $\mathrm{B}$ modes have very similar shape and amplitude. The maximum signal is obtained for an angular scale of 25 arcmin, which physically corresponds (because the aperture mass probes smaller scales) to the chip size of 7 arcmin. This is because the PSF changes from chip to chip.

produced wings at the edge of the CCDs. A second order fitting removed most of the unwanted $B$ mode contribution without spoiling the $E$ mode signal (as also found by Massey et al. 2001). As shown in Fig. 1, the residual systematics are consistent with zero up to 10 arcmin and remains flat over the whole angular scale explored by the data. Clearly, the signal is dominated by the $E$ mode contribution at least up to $25 \mathrm{arcmin}$. This demonstrates that a signal produced by intrinsic alignment of galaxies is not detected at this level. We also computed the $E$ and $B$ modes on the stars before the PSF correction. The result is shown in Fig. 2, and explicitly demonstrates that the amount of systematic errors is almost equally distributed between the two channels and that it is unlikely this will change by the PSF correction process. However, it is important to note that, for the largest scales, given the small statistical errors, the difference between the $E$ and $B$ modes could be very significant. This means, in the case of a residual $B$ mode (like ours), that a $E$ mode cleaning based on a $B$ mode subtraction could potentially lead to a biased signal estimate. We argue here that the safest action against a residual $B$ mode is to use this residual signal to enlarge the $E$ mode measurements, as will be described in Sect. 4.2 .

\section{Parameter estimation}

\subsection{Redshift distribution of galaxies in the VIRMOS-DESCART data}

We estimated the redshift distribution of our catalogue from a combination of the Hubble Deep Fields North and South data (Fernández-Soto et al. 1999; Chen et al. 1998) and VLT observations of the cluster MS 1008-1224. Both HDF and MS 10081224 observations are much deeper than the limiting magnitude of cosmic shear sample of galaxies considered in this work $\left(I_{\mathrm{AB}}=24.5\right)$, so that magnitude measurements and photometric redshift estimates up to $I_{\mathrm{AB}}=24.5$ are based on reliable data with high signal-to-noise ratio. Although its effect is negligible, we also took into account the weight-magnitude relation (the fact that fainter galaxies have statistically a smaller weight) in the redshift distribution estimation.

The VLT MS 1008-1224 galaxy sample comprises deep $U B V R I$ observations, carried out by the Science Verification Team (SVT) at ESO/VLT with the FORS1 and FORS2 instruments (Appenzeller et al. 1998) and deep $J$ and $K$ data obtained at the ESO/NTT with SOFI (Program 66.A-0316(A); PI Mellier). The extension of early deep SVT observations to U band with FORS2 and more recently to near infrared with SOFI, which has similar field of view as FORS (5.5 arcmin against 6.8 arcmin), allows us to considerably improve the accuracy of photometric redshifts of foreground, cluster and background galaxies over the whole field. In comparison with the HDF, the VLT/NTT observations are not as deep, but they provide a much larger sample of galaxies because they cover of field of view 15 times larger than HDF. In total, 920 galaxies with $I_{\mathrm{AB}} \leq 24.5$ and $U B V R I J K$ data have been added to HDF data.

The deep $U B V R I$ data are described at the ESO site ${ }^{5}$ and in Athreya et al. (2002). A complete description of the new $J$ and $K$ band data will be presented elsewhere (Gavazzi et al. in preparation). Briefly, the exposure times of NTT/SOFI $J$ and $K$ bands were $5 \mathrm{~h} 30$ and $6 \mathrm{~h}$ respectively. The completeness limits are $J=23$. and $K=22$. and both $J$ and $K$ complete samples comprise more than $90 \%$ of the $I_{\mathrm{AB}} \leq 24.5$ galaxies. Hence, most galaxies used to determine the photo- $z$ distribution of galaxies up to $I_{\mathrm{AB}} \leq 24.5$ have reliable $J$ and $K$ photometric measurements. We are able to secure a collection of redshifts on a very large sample of galaxies, covering a broad magnitude range and uniformly distributed over the whole field. The presence of the lensing cluster $(z=0.306)$ in the field only affects the redshift range $0.26<z<0.36$. These data have been removed from the sample and the redshift distribution interpolated in this redshift range. The magnification bias may also change the redshift distribution of galaxies inside the very center of the cluster where the gravitational depletion is significant (see Athreya et al. 2002). We therefore also removed the central part $(R<40$ arcsec $)$ of the cluster from the sample. Since this region is also the most contaminated by the brightest cluster members, the depletion itself turns out to have no impact on the galaxy selection criterion.

5 http://www.hq.eso.org/science/ut1sv 
The photometric redshifts $\left(z_{\text {phot }}\right)$ were measured using the fitting algorithm hyperz developed by Bolzonella et al. (2000). Each $z_{\text {phot }}$ is inferred by comparing the spectral energy distribution of galaxies, as sampled by their UBVRIJK photometric flux, to a set of spectral templates representative of common late and early type galaxies which are followed with look-back time according to Bruzual \& Charlot's evolution models (GISSEL98; Bruzual \& Charlot 1993). The validation of hyperz is discussed at length in Bolzonella et al. (2000). It has been conclusively gauged against spectroscopic redshift on MS 1008-1224 data by Athreya et al. (2002). Details on photometric redshift techniques can be found in those papers.

The compiled photometric distribution is shown in Fig. 3. For the purpose of marginalisation we parameterise this distribution with the following normalised function:

$n(z)=\frac{\beta}{z_{\mathrm{s}} \Gamma\left(\frac{1+\alpha}{\beta}\right)}\left(\frac{z}{z_{\mathrm{s}}}\right)^{\alpha} \exp \left[-\left(\frac{z}{z_{\mathrm{s}}}\right)^{\beta}\right]$,

where $\alpha=2$ and $\beta=1.2$. For these values of $\alpha$ and $\beta$, the mean redshift is $\bar{z}_{\mathrm{s}} \approx 2.1 z_{\mathrm{s}}$ and the median redshift is $\approx 1.9 z_{\mathrm{s}}$. We allowed $z_{\mathrm{s}}$ to vary from 0.24 to 0.64 , which corresponds to a mean redshift varying from 0.5 to 1.32 . These two extreme models are shown in Fig. 3: they are clearly conservative bounds on the redshift distribution in the data. The curve in Fig. 3 shows the best fit model, with $z_{\mathrm{s}}=0.44\left(\bar{z}_{\mathrm{s}}=0.9\right)$.

\subsection{Maximum likelihood}

The dominant cosmological parameters for the 2-point cosmic shear statistics are the mean mass density $\Omega_{\mathrm{M}}$, the power spectrum normalisation $\sigma_{8}$, the shape parameter $\Gamma$ and the redshift of the sources (see Bernardeau et al. 1997; Jain \& Seljak 1997; van Waerbeke et al. 1999) Our parameter space has therefore four dimensions, but we truncate the exploration volume to a realistic range defined as $\Omega_{\mathrm{M}} \in[0.1,1], \sigma_{8} \in[0.3,1.6]$ and $\Gamma \in[0.05,0.7]$ with a sampling of $10 \times 14 \times 14$. For the analysis of the VIRMOS-DESCART data, the redshift of sources is parameterised by Eq. (13) with $z_{s} \in[0.24,0.64]$ and a sampling of 9 . For the simulations, the sources are placed at redshift unity, therefore in the maximum likelihood analysis we assumed we knew the shape (Dirac distribution), but we allowed the redshift $z_{\mathrm{s}}$ to vary between 0.7 and 1.2 (sampling of 6). In fact we found that the real shape of the source distribution does not matter, but the agreement with the mean redshift does. This parameter range box $\left(\Omega_{\mathrm{M}}, \Gamma, \sigma_{8}, z_{\mathrm{s}}\right)$ defines what we call the default prior box. The model predictions are then interpolated with an oversampling seven times higher in each dimension.

Let $d_{i}$ be the data vector (i.e. the aperture mass $\left\langle M_{\mathrm{ap}}^{2}\right\rangle$ for different scales $\left.\theta_{i}\right)$, and $m_{i}\left(\Omega_{\mathrm{M}}, \sigma_{8}, \Gamma, z_{\mathrm{s}}\right)$ the model predictions. The likelihood function of the data is

$\mathcal{L}=\frac{1}{(2 \pi)^{n}|\mathbf{C}|^{1 / 2}} \exp \left[\left(d_{i}-m_{i}\right) \mathbf{C}^{-1}\left(d_{i}-m_{i}\right)^{\mathrm{T}}\right]$,

where $n=10$ is the number of scales and $\mathbf{C}$ is the $10 \times 10$ covariance matrix,

$C_{i j}=\left\langle\left(d_{i}-m_{i}\right)^{\mathrm{T}}\left(d_{j}-m_{j}\right)\right\rangle$.

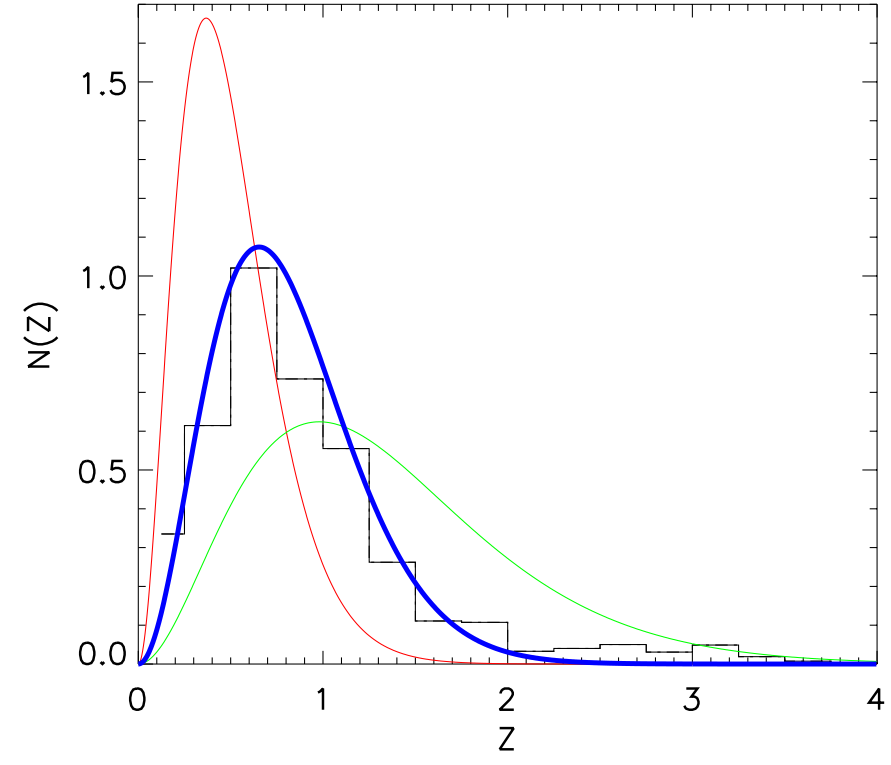

Fig. 3. The histogram shows the photometric redshift distribution from the joint VLT and HDF fields. The thick solid line is the theoretical $n(z)$ from Eq. (13) with $z_{\mathrm{s}}=0.44$. The low and high redshift thin solid lines correspond to $z_{\mathrm{s}}=0.24$ and $z_{\mathrm{s}}=0.64$, our extreme redshift distributions used in this paper.

C can be decomposed as $\mathbf{C}=\mathbf{C}_{\mathrm{n}}+\mathbf{C}_{\mathrm{s}}+\mathbf{C}_{\mathrm{b}}$, where $\mathbf{C}_{\mathrm{n}}$ is the statistical noise, $\mathbf{C}_{\mathrm{s}}$ the cosmic variance covariance matrix and $\mathbf{C}_{\mathrm{b}}$ the residual systematics bias. $\mathbf{C}_{\mathrm{n}}$ has been measured using Eq. (9) of Pen et al. (2002), so we just reproduce here its general behaviour: the top panel in Fig. 4 shows the crosscorrelation coefficient for 2,10 , and 35 arcmin with the other scales. In order to account for residual systematics, we decided to add quadratically the residual $B$ mode (see the bottom panel in Fig. 1) to the error of the signal. Given that there is no clearly identified scheme to deal with the residual systematics yet, this appears to be the safest and most conservative attitude. The diagonal part of the bias correlation matrix $\mathbf{C}_{\mathrm{b}}$ is therefore given by the $B$ mode signal, and the off-diagonal terms follow the same correlation properties as the $E$ mode (the $E$ and $B$ covariance matrices for the statistical noise are actually identical).

The cosmic variance covariance matrix $\mathbf{C}_{\mathrm{s}}$ is trickier to estimate. Assuming the field is Gaussian is too simplistic, since the observed scales are within the non-linear and weakly nonlinear regimes, so in principle a complete description of nonGaussian contributions to the error budget cannot be carried out without detailed cosmological simulations. In order to avoid this involved procedure, we focused instead on a simpler alternative based on non-linear perturbation theory. It was pointed out in Scoccimarro et al. (1999) that, for the convergence power spectrum, the ratio of the Gaussian to non-Gaussian errors is almost independent of scale, and close to 1 for any cosmology. We investigated whether this statement could be also valid in real space, using three ray-tracing simulations for three different cosmological models (Jain et al. 2000). Figure 5 shows this ratio for $\Lambda \mathrm{CDM}, \tau \mathrm{CDM}$ and OCDM cluster normalised models. For scales larger than $3 \mathrm{arcmin}$, it is indeed nearly independent of cosmology. At smaller scales the $\Lambda \mathrm{CDM}$ model has a larger cosmic variance, but this is not important, since 

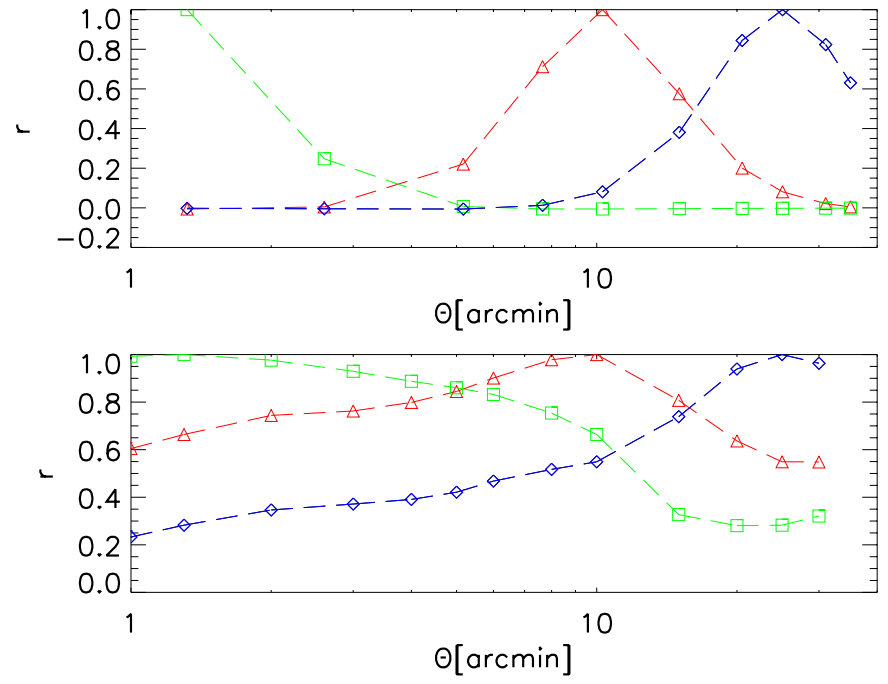

Fig. 4. Cross-correlation coefficient $r\left(\theta, \theta^{\prime}\right)$ as a function of scale $\theta$ for three different scales $\theta^{\prime}$ for the statistical noise (above) and the cosmic variance (below). The three scales are 1.3' (squares), 10.3' (triangles) and 25.1' (diamonds). The cosmic variance cross-correlation is obtained from ray-tracing simulations (Jain et al. 2000).

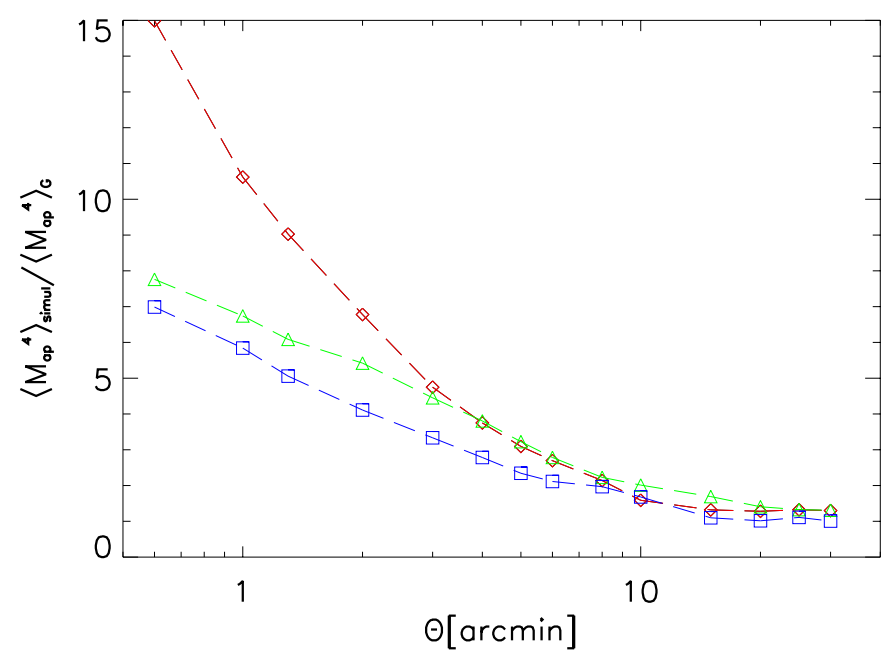

Fig. 5. Ratio between the aperture mass dispersion obtained from ray-tracing simulations (Jain et al. 2000) and the dispersion obtained from the Gaussian field hypothesis for a survey of similar size. The three curves correspond to a $\tau \mathrm{CDM}$ (squares), OCDM (triangles) and $\Lambda \mathrm{CDM}$ (diamonds), showing that the ratio is little dependent of the cosmological model above $3^{\prime}$.

below a few arcminutes statistical noise dominates (see Fig. 10). Therefore, although the result in Scoccimarro et al. (1999) is clearly not valid for very small scales, it is still weakly sensitive to cosmology. We then approximated $\mathbf{C}_{\mathrm{s}}$ in the following way: we compute the Gaussian cosmic variance for each model, then we convert it to a non-Gaussian cosmic variance using the correction factor in Fig. 5. The cross-correlation coefficient is also measured from the ray-tracing simulations. The different scales are somewhat correlated, as shown in Fig. 4 (bottom panel). As we shall see in Fig. 10, even an incorrect estimate of the cosmic variance by a factor of two has no consequences on our parameter estimate, given that the errors are dominated by $\mathbf{C}_{\mathrm{n}}$ and $\mathbf{C}_{\mathrm{b}}$.

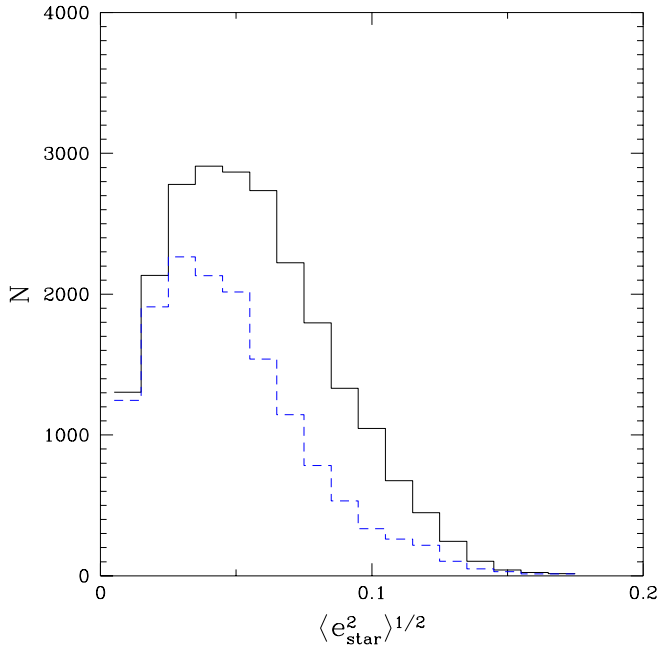

Fig. 6. Histogram of the Point Spread Function anisotropy of the stars in the simulated images (solid line) and in the VIRMOS-DESCART survey (dashed line).

\section{Applications}

We now apply the likelihood analysis to simulated sky images and to the VIRMOS-DESCART data.

\subsection{Mock catalogues}

The mock catalogues are generated from simulated sky images following the procedure described in Erben et al. (2001) in which a simulated catalogue of galaxies is first lensed and then used to generate a CCD image of the sky. But instead of having a constant shear amplitude on each field, the distortion of galaxies is introduced using ray-tracing simulations (Jain et al. 2000). We used the two ray-tracing simulations presented in Jain et al. (2000): one is OCDM, as described in Sect. 3, and the other is a $\tau \mathrm{CDM}$ with $\Gamma=0.21$ and $\Omega=1$. For each simulation we produced 11 square degrees of simulated sky images in blocks of $6.7 \times 6.7$ arcmin containing roughly 30 galaxies per $\operatorname{arcmin}^{2}$, with a pixel size of 0.2 arcsec. As in real sky surveys, the mock catalogues contain the following features

- galaxy intrinsic shape fluctuations;

- masks;

- noise from galaxy shape measurements (which includes pixel noise, and noise induced by our shape correction process) and systematics from PSF corrections,

and the simulated images reproduce similar observational conditions as real data (PSF anisotropy, limiting magnitude, luminosity functions, galaxy and star number densities, intrinsic ellipticity...). The PSF is constant in amplitude and orientation over blocks of $6.7 \times 6.7 \mathrm{arcmin}$, it is randomly chosen between blocks. The simulated galaxies are then analysed exactly in the same way as real data, following the procedure described in (Van Waerbeke et al. 2000, 2001b), in particular, the PSF fitting on the stars was performed on the individual chips of the simulation.

Figure 6 compare the star anisotropy between the simulated fields (solid line) and the data (dashed line). The likelihood 

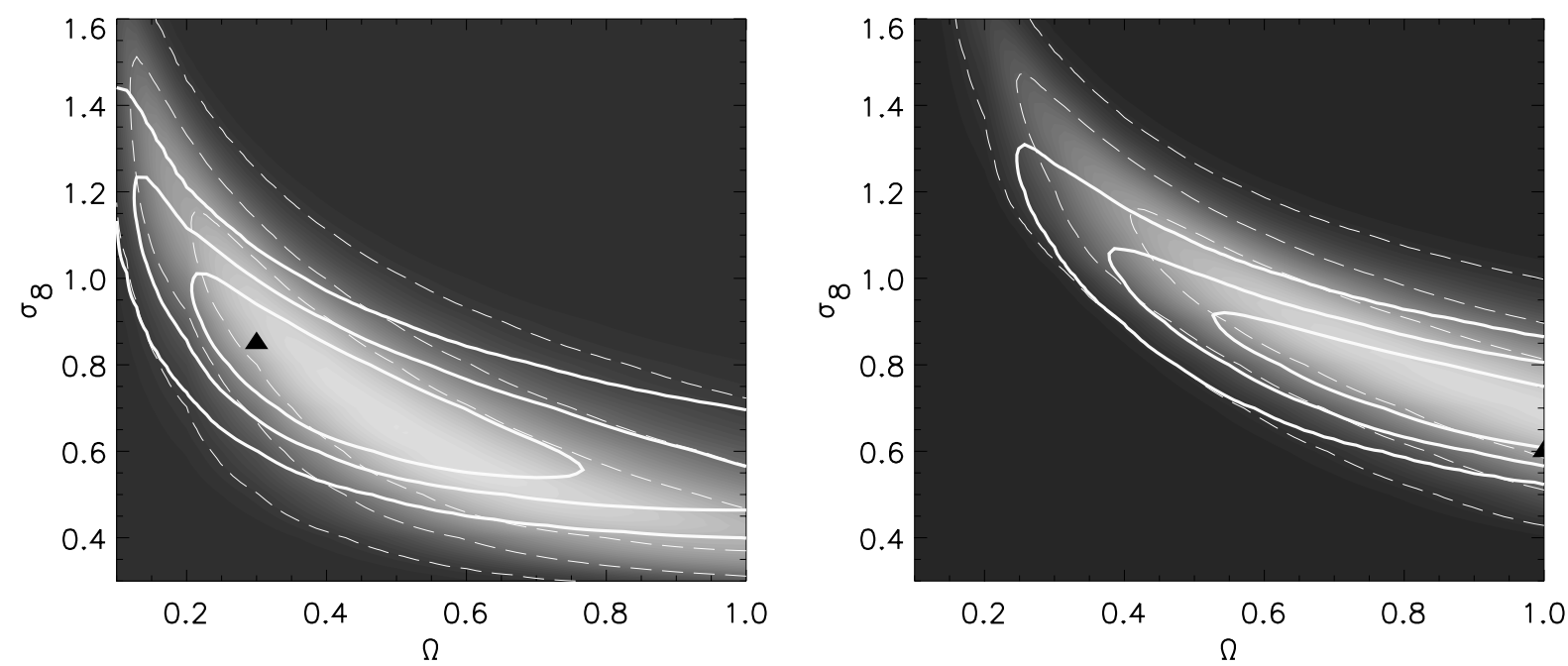

Fig. 7. Constraints on $\sigma_{8}$ and $\Omega_{\mathrm{M}}$ for the OCDM (left) and $\tau$ CDM (right) simulations. The gray levels and the dashed contours show the simulation default prior constraints, with confidence levels of $0.68,0.95$ and 0.999 . The true model, indicated by a dark triangle, is $\Omega=0.3$, $\sigma_{8}=0.85$ (left) and $\Omega=1, \sigma_{8}=0.6$ (right). The thick solid line contours are for a prior $\Gamma \in[0.1,0.3]$ and $\bar{z}_{\mathrm{s}} \in[0.9,1.1]$.

function is computed for 11760 models $(10 \times 14 \times 14 \times 6)$. Figure 7 shows the results for the maximum likelihood analysis of these two simulation sets. Note that we display two types of contours: the dashed contours shows the constraints with the marginalisation on $\Gamma$ and $z_{\mathrm{s}}$ done over the default-prior box, and the solid line the constraints obtained with a more realistic marginalisation. We clearly converge to the right cosmological model, which validates our likelihood approach for the data (see Sect. 5.2). However, we should emphasise that the underlying assumption of the maximum likelihood method is that the theoretical models used are an accurate representation of the real world. Our simulations shows this is unfortunately not necessarily the case with today's lensing data sets. For instance, it was shown in Van Waerbeke et al. (2001a) (Fig. 2) that the non-linear predictions fail badly for the aperture mass with a $\tau \mathrm{CDM}$ model. This failure should not be surprising: it was already noticed in the projected power spectrum in Jain et al. (2000) (Fig. 8), and even the VIRGO simulations (see Jenkins et al. 1998, Fig. 7) demonstrated a mismatch between the 3D non-linear predictions and the measured power spectrum. In the case of our $\tau \mathrm{CDM}$ simulation, the potential problem is an overestimate of the power spectrum normalisation $\sigma_{8}$. This is illustrated in Fig. 8 where we compare the measured power to the Peacock \& Dodds prediction for that model. With a smaller statistical error and/or residual bias and cosmic variance, the true model with $\sigma_{8}=0.6$ will become excluded from our $3-\sigma$ contours in the right panel of Fig. 7. In fact a maximum likelihood analysis on the noise free catalogue would give $\sigma_{8}=0.8$, that is $20 \%$ larger than the true $\sigma_{8}$, which corresponds to the lack of power in the predicted non-linear signal. We will present a more detailed discussion of this problem in Sect. 6 .

\subsection{VIRMOS-DESCART data}

We first consider flat cosmologies, since this is the class of models currently favoured by the cosmic microwave background measurements (de Bernardis et al. 2000), but alternative

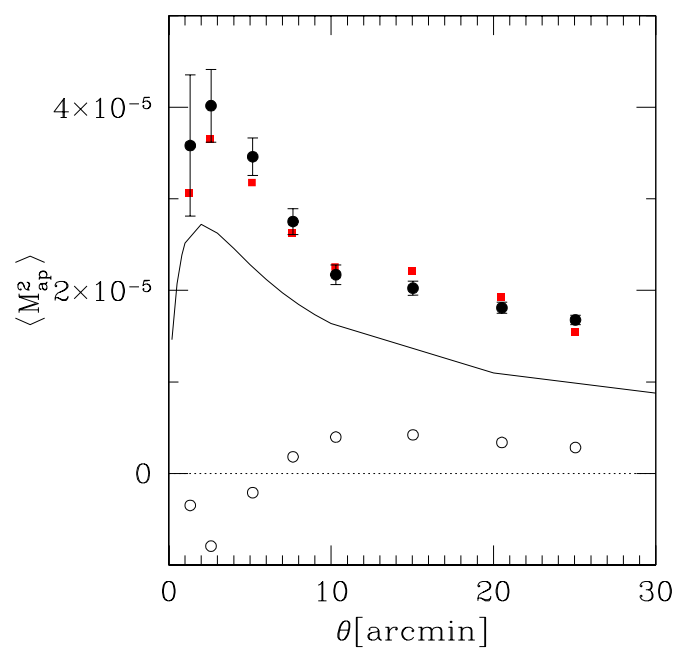

Fig. 8. The filled circles with error bars show the aperture mass measured on the $\tau \mathrm{CDM}$ simulated sky images, while the filled small squares show the signal measured in the input catalogue. The open circles show the measured residual $B$-mode. Measurements and simulation are in perfect agreement, but the non-linear prediction obtained from Peacock \& Dodds (1996) for this model (solid line) is significantly off.

open universes are also investigated. In either case, the likelihood function is computed for $17640(10 \times 14 \times 14 \times 9)$ models using Eq. (8), as a function of angular scale, and for a regular spacing in the default prior box.

Figure 9 shows the four-parameter constraints for different priors and marginalised parameters for the flat cosmology. The dashed lines shows the $68 \%, 95 \%$ and $99.9 \%$ contours when the default prior is applied for the two remaining parameters. We cannot extract strong constraints in this case, but the right panel shows an interesting correlation between $\Gamma$ and $z_{\mathrm{s}}$ : a flat power spectrum (large $\Gamma$ ) can account for an underestimated source redshift. The thick solid curves shows the same contours with a stronger prior: $\Gamma$ and $z_{\mathrm{s}}$ are marginalised over [0.1,0.4] and 

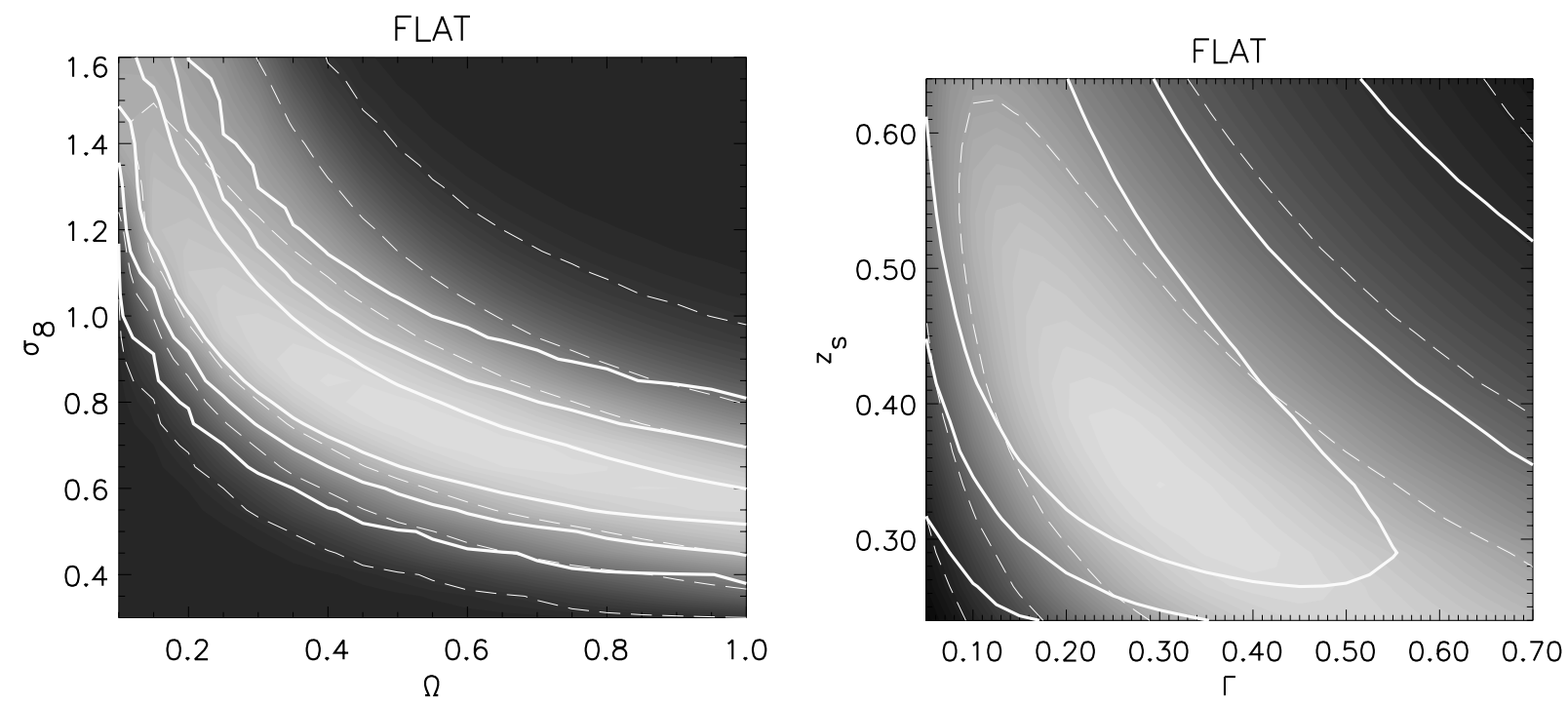

Fig. 9. Left: constraints on $\Omega$ and $\sigma_{8}$ for the flat cosmologies. The confidence levels are $[68,95,99.9]$ from the brightest to the darkest area. The galaxy sample comprises objects with $I_{\mathrm{AB}}>21$. The gray area and the dashed contours correspond to the contours computed with a full marginalisation over the default prior $\Gamma \in[0.05,0.7]$ and $z_{\mathrm{s}} \in[0.24,0.64]$. The thick solid line contours are obtained from the prior $\Gamma \in[0.1,0.4]$ and $z_{\mathrm{s}} \in[0.39,0.54]$ (which is a mean redshift $\bar{z}_{\mathrm{s}} \in[0.8,1.1]$ ). Right: constraints on $\Gamma$ and $\Omega_{\mathrm{M}}$ for the flat cosmologies. The contours have the same statistical meaning as in the left panel, but in this case the dashed lines correspond to a marginalisation over the default prior $\Omega \in[0.1,1]$ and $\sigma_{8} \in[0.3,1.6]$, and the thick lines for $\Omega \in[0.1,0.4]$ and $\sigma_{8} \in[0.7,1.3]$.

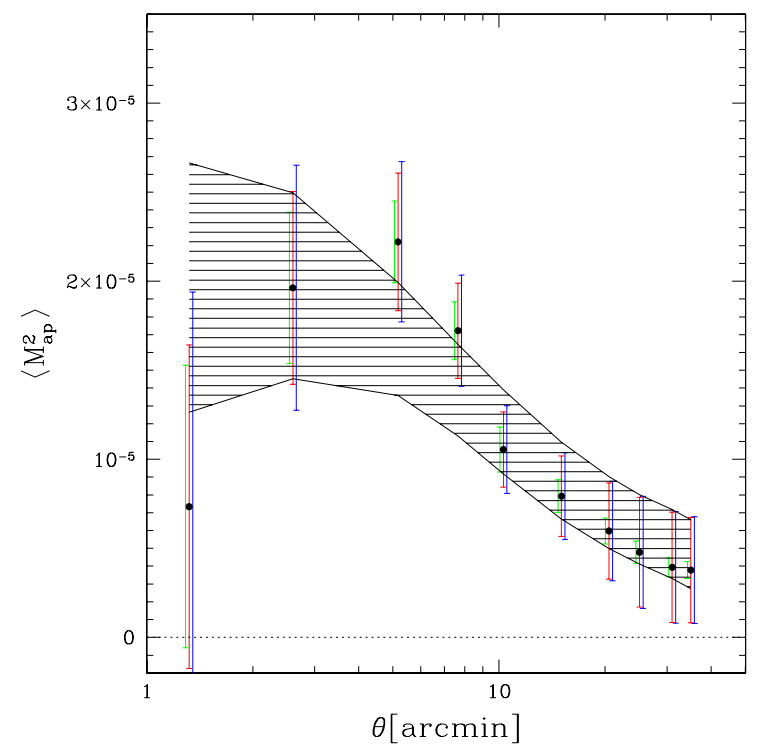

Fig. 10. The aperture mass statistic $\left\langle M_{\mathrm{ap}}^{2}\right\rangle$ measured on the data (see Fig. 1) compared to all the models included in the $68 \%$ contour (shaded area). For each measurement point, the error bars from left to right are: statistical errors, statistical error and residual bias, statistical errors and bias and cosmic variance.

$[0.39,0.54]$ for the left panel, and $\Omega_{\mathrm{M}}$ and $\sigma_{8}$ are marginalised over $[0.1,0.4]$ and $[0.7,1.3]$ for the right panel. We obtain the following constraint from the left panel:

$\sigma_{8}=(0.57 \pm 0.04) \Omega_{\mathrm{M}}^{(0.24 \mp 0.18) \Omega_{\mathrm{M}}-0.49}$,

for the $68 \%$ level and

$\sigma_{8}=(0.58 \pm 0.13) \Omega_{\mathrm{M}}^{(0.205 \mp 0.025) \Omega_{\mathrm{M}}-0.48}$ for the $95 \%$ contour. Constraints on the shape of the mass power spectrum can be derived from the right panel if one assumes that photometric redshifts provide the exact redshift distribution (which is given by $z_{\mathrm{s}}=0.44$ ). In that case we have $\Gamma \in[0.12,0.38]$.

Figure 10 shows the aperture mass measurements with all the models inside the $68 \%$ contours as the shaded area. The error bars show the contribution the three errors as a function of scale. Each set of errors shows 3 bars, which from left to right are: statistical noise, bias added, cosmic variance added. We see that the statistical noise dominates at small scales, wheras the systematic residuals dominate at larger scales. Importantly, the cosmic variance is never the most important contribution.

It is interesting at this stage to compare our results with measurements from other surveys. A comparison with Cosmic Microwave Background (CMB) constraints reveals that weak lensing will be helpful to break the degeneracy between $\sigma_{8}$ and $\Omega_{\mathrm{M}}$. Recently, Lahav et al. (2001) have shown CMB estimation of these two parameters, assuming that the Hubble constant is a Gaussian variable centred at $h=0.7$ with an rms of 0.07 , and fixing other parameters (primordial spectral index $n=1$, baryon density $\Omega_{\mathrm{b}}=0.02$ and reonization depth $\tau=0$ ). Their results are shown as solid lines in Fig. 12. An overlay of our constraints on the same plot (dashed lines) show that a combination of CMB and lensing would favour low density models $\left(\Omega_{\mathrm{M}} \sim 0.3-0.4\right)$ and rather low normalisation $\left(\sigma_{8} \sim 0.7-0.8\right)$. This plot reveals that the lensing constraints are almost orthogonal to the CMB constraints. As explained in Lahav et al. (2001), a weaker prior on $h$ would extend the CMB contours and restore the degeneracy between $\Omega_{\mathrm{M}}$ and $\sigma_{8}$, making $\Omega_{\mathrm{M}}=1$ a viable solution again. But we see that lensing rules out such a solution because $\Omega_{\mathrm{M}} \sim 1$ with $\sigma_{8}>0.8$ is excluded. Given that CMB alone predicts a flat Universe, the 


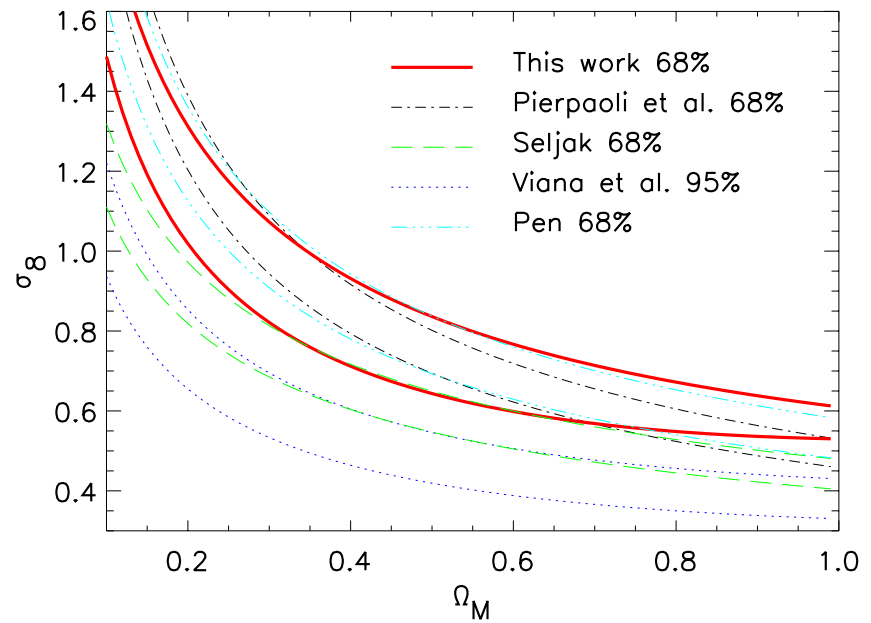

Fig. 11. The $\Omega-\sigma_{8}$ constraints for a flat universe from our work, compared to the cluster normalisation constraints.

inconsistency between CMB and lensing for $\Omega_{\mathrm{M}}=1$ should be interpreted as in favour of a non-zero cosmological constant. The fact that $\mathrm{CMB}$ and lensing have orthogonal constraints in the $\left(\sigma_{8}, \Omega_{\mathrm{M}}\right)$ parameter space make them indeed very complementary. A complete analysis which takes into account the marginalisation over the other parameters (baryon density, $\tau$, etc.) is in progress. However, we should note the area of agreement between our results and the combined $\mathrm{CMB}+2 \mathrm{dF}$ constraints (Lahav et al. 2001, Fig. 5).

We should also compare our results more closely to the cluster normalisation constraints, since those two methods are expected to probe a similar combination of $\sigma_{8}$ and $\Omega_{\mathrm{M}}$. Figure 11 shows our results and those obtained from cluster measurements. As it was claimed before (Maoli et al. 2001; Van Waerbeke et al. 2001b; Rhodes et al. 2001), a joint estimate of $\Omega_{\mathrm{M}}$ and $\sigma_{8}$ from weak lensing is consistent with the former cluster abundance estimates (Pen 1998; Pierpaoli et al. 2001). Recently, these estimates were revisited but the new results are puzzling (see Fig. 11): whereas Seljak (2001) is marginally consistent with our constraints, on the other hand, Viana et al. (2001) is significantly lower. Note also that Borgani et al. (1999) already pointed to a lower normalisation two years ago. Pen (1998) performed direct hydrodynamic simulations to predict the cluster X-ray temperature function for various cosmological models. This bypasses the difficult mass ladder of converting $\mathrm{N}$-body or Press-Schechter mass functions into a temperature function, and/or accounting for scatter in this relation, and the results are in good agreement with the cosmic shear constraints. However, some effects that may still not be accounted for in simulations include non-gravitational feedback from galaxies, magnetic fields, thermal conduction. These effects may all limit the intrinsic accuracy of cluster normalisations. We will not enter into the debate between the cluster estimates here, but if the low normalisation is confirmed, this discrepancy might be an important finding: it might be an indication of the inaccuracy of the non-linear predictions, as shown in Sect. 5.1.

The maximum likelihood analysis was also carried out for open cosmologies. The probability contours shown in Fig. 13

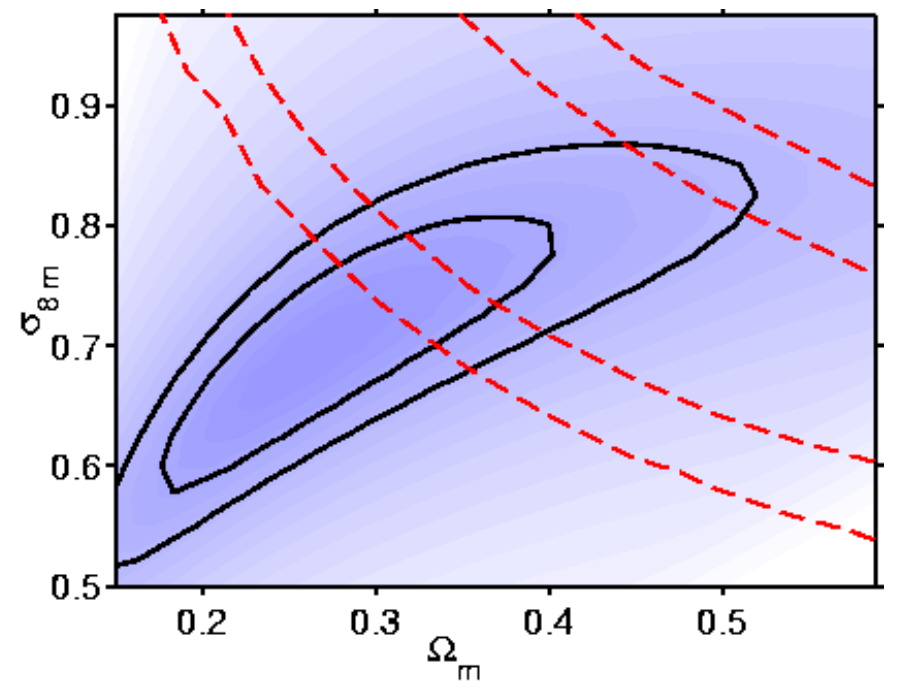

Fig. 12. Solid line: the CMB alone constraints as described in Lahav et al. (2001) (figure done by S. Bridle). The CMB priors are given by a concordance model, with the Hubble constant $h=0.7$ with an rms of 0.07 , the baryon density $\Omega_{\mathrm{b}}=0.02$, the primordial spectral index $n=1$, and the reonization depth $\tau=0$. Dashed lines are our cosmic shear constraints for the flat cosmologies (see Fig. 9). In either case, the contours show the $68 \%$ and $95 \%$ confidence levels.

summarise the results which are indeed similar to the flat case. However, low density $\left(\Omega_{\mathrm{M}}<0.2\right)$, open universes, seem more difficult to reconcile with the data than flat models. This contradiction between observations and low density open universe results mostly from the small scale measurement of $\left\langle M_{\mathrm{ap}}^{2}\right\rangle$ (Fig. 10). Indeed, low density open universes predict too much power at small scale as compared to what can be allowed from the amplitude of $\left\langle M_{\mathrm{ap}}^{2}\right\rangle$ on scale of about one arcmin. This clear difference between open and flat $\Lambda$ CDM universes was already pointed out by Schneider et al. (1998) (Fig. 3) but this is the first time that it has been seen in observations.

Finally, although the uncertainties are still large enough to leave room for a large sample of models, it is interesting to show how cosmic shear data can be used jointly with several independent surveys (not only CMB). Because weak lensing analyses directly probe the dark matter distribution, cosmic shear surveys provide the best way to constrain $\sigma_{8}$. It is then relevant to focus on this parameter, using values of other cosmological parameters as they are derived from external data sets. Assuming the mean redshift of sources is $z_{\mathrm{s}}=0.9$ and $h=0.7 \pm 0.1$ (Freedman et al. 2001, from the HST Key Project), a flat universe (from CMB data) with a baryon fraction inferred from BBN and $\Gamma=0.2 \pm 0.05$ (Szalay et al. 2002, from the SDSS redshift survey), we then have $\Omega_{\mathrm{M}} \approx \Gamma / h \approx 0.3 \pm 0.1$. In that case, the VIRMOS-DESCART cosmic shear survey provide $\sigma_{8}=0.98 \pm 0.06$, in good agreement with other independent methods. As compared to the joint CMB-cosmic shear alone discussed previously, the normalisation is higher. This is mainly due to the low $\Omega_{\mathrm{M}}$ (i.e. high $\sigma_{8}$ ) combined with a strong prior on $\Gamma$ (inferred from the galaxy redshift survey) and on the source redshift. 


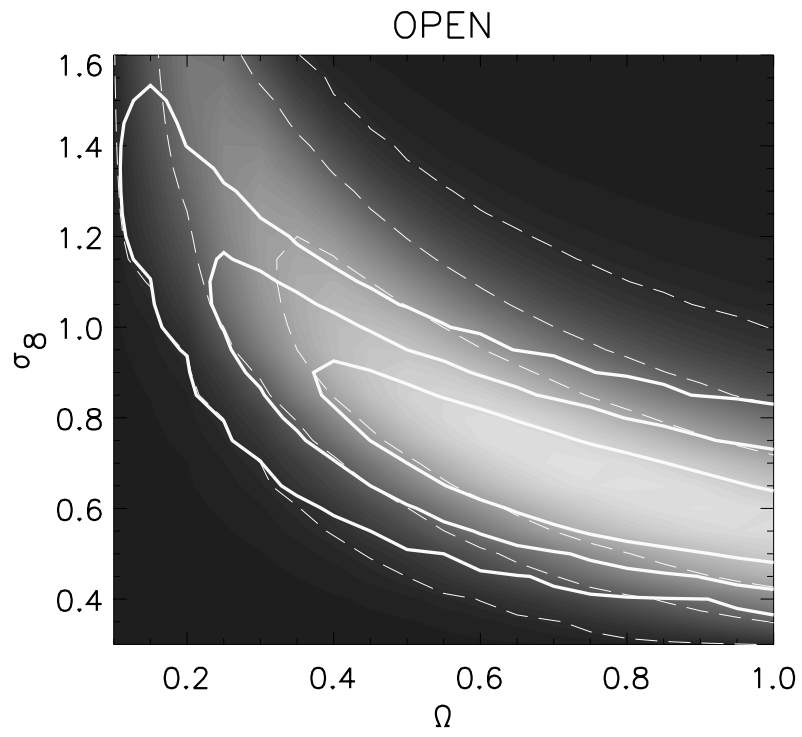

Fig. 13. Same as Fig. 9 for the open cosmologies.

\section{Conclusion}

We explored a 4-dimensional parameter space using the most recent cosmic shear data. We included all possible sources of error: statistical noise, cosmic variance and residual systematics. We obtained constraints on $\Omega_{\mathrm{M}}$, the power spectrum slope $\Gamma$, its normalization $\sigma_{8}$ and the redshift of the sources $z_{\mathrm{s}}$. We marginalized over $\Gamma$ and $z_{\mathrm{s}}$. Both the marginalisation, and the inclusion of all the sources of error, make our results for $\left(\Omega_{\mathrm{M}}, \sigma_{8}\right)$ robust. We pointed out the complementarity between cosmic shear and $\mathrm{CMB}$ measurements for breaking the degeneracy among $\Omega_{\mathrm{M}}$ and $\sigma_{8}$, and the good agreement with $\mathrm{CMB}$ and $\mathrm{CMB}+2 \mathrm{dF}$ constraints. However, our results are only in marginal agreement with the latest cluster abundance constraints, which give a lower normalization $\sigma_{8} \sim 0.7$ for $\Omega_{\mathrm{M}} \sim 0.3$. If this discrepancy is confirmed in either measurements, this could be interpreted as an indication that the lensing non-linear prediction is not accurate enough given the already small size of the cosmic shear errors. This interpretation is supported by ray-tracing simulations in a $\tau$ CDM model, and more generaly by a comparison of the VIRGO simulations with the Peacock \& Dodds non-linear prescription. It was claimed a $15 \%$ accuracy in their original paper (Peacock \& Dodds 1996), although it might be a bit more for some cosmological models (Jenkins et al. 1998). This is clearly the maximum uncertainty we can tolerate with today's lensing measurements, and it will be insufficient for forthcoming surveys. This potential problem suggests three paths for improvements:

1. More extensive use should be made of ray-tracing simulations to test predictions at non-linear scales. Until now, most ray-tracing simulations have assumed that all sources are at a single redshift $\left(z_{\mathrm{s}}=1\right)$. However, the source redshift distribution depends on how structures grow in the Universe, and any realistic simulations must take this into account.

2. Theoretical predictions should be improved. There might be some hope by reviving the halo models which give

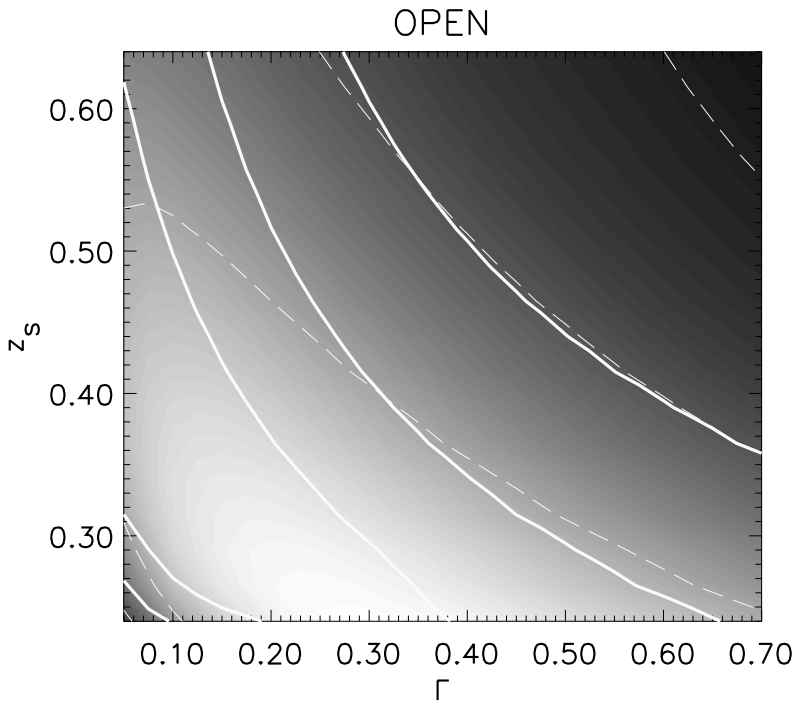

predictions close, but not identical, to Peacock \& Dodds (like the peakpatch approach, Bond, priv. comm.).

3. Ultimately, cosmic shear observations will lead to a measurement of the 3D mass power spectrum in a nonparametric way, and therefore solve all the problems associated with non-linear modeling. This is possible only if the cosmological parameters are determined by other means. For instance, the linear mass power spectrum and cosmological parameter measurements at large scales using combined (or not) lensing data with cosmic microwave background or X-rays could be obtained, and used to deconvolve the non-linear power spectrum. This means that we will be able to deconvolve the projected mass power spectrum measured from cosmic shear observations and recover the true $3 \mathrm{D}$ power spectrum. This is a work in progress, in which we are trying to recover the galaxygalaxy and galaxy-mass correlations as well, using tomography techniques (Hu 1999).

Acknowledgements. We thank Dmitri Pogosyan and Carlo Contaldi for useful discussions concerning maximum likelihood techniques and Peter Schneider and Henk Hoekstra for discussions and comments on the manuscript. Discussions with Roman Scoccimarro on the Peacock $\&$ Dodds prescription were also very useful. We are grateful to Uros Seljak and the referee Alexandre Réfrégier whose comments and criticisms lead to improve the manuscript. We thank the VIRMOS and Terapix teams who obtained and processed the VIRMOS-DESCART data. This work was supported by the TMR Network "Gravitational Lensing: New Constraints on Cosmology and the Distribution of Dark Matter" of the EC under contract No. ERBFMRX-CT97-0172. YM thanks CITA for hospitality.

\section{References}

Appenzeller, I., Fricke, K., Furtig, W., et al. 1998, The Messenger, 94, 1

Athreya, R., Mellier, Y., van Waerbeke, L., et al. 2002, A\&A, 384, 743

Bacon, D. J., Refregier, A. R., \& Ellis, R. S. 2000, MNRAS, 318, 625 
Bernardeau, F., van Waerbeke, L., \& Mellier, Y. 1997, A\&A, 322, 1 Bolzonella, M., Miralles, J., \& Pelló, R. 2000, A\&A, 363, 476 Borgani, S., Rosati, P., Tozzi, P., \& Norman, C., 1999, ApJ, 517, 40 Bruzual, A., \& Charlot, S. 1993, ApJ, 405, 538

Chen, H., Fernandez-soto, A., Lanzetta, K. M., et al. 1998, in Photometry and Photometric Redshifts of Galaxies in the Hubble Deep Field South Nicmos Field [astro-ph/9812339]

Crittenden, R. G., Natarajan, P., Pen, U., \& Theuns, T. 2002, ApJ, 568, 20

de Bernardis, P., Ade, P. A. R., Bock, J. J., et al. 2000, Nature, 404, 955

Erben, T., Van Waerbeke, L., Bertin, E., Mellier, Y., \& Schneider, P. 2001, A\&A, 366, 717

Fernández-Soto, A., Lanzetta, K. M., \& Yahil, A. 1999, ApJ, 513, 34

Freedman, W., Madore, B., Gibson, B., et al. 2001, ApJ, 553, 47

Haemmerle, H., Miralles, J. M., Schneider, P., et al. 2002, A\&A, 385, 743

Hoekstra, H., Yee, H. K. C., \& Gladders, M. D. 2001, in Where's the matter? Tracing dark and and bright matter with the new generation of large scale surveys, Marseille [astro-ph/0109514]

Hoekstra, H., Yee, H. K. C., Gladders, M. D., et al. 2002, ApJ, in press [astro-ph/0202285]

$\mathrm{Hu}$, W. 1999, ApJ, 522, L21

Jain, B., \& Seljak, U. 1997, ApJ, 484, 560

Jain, B., Seljak, U., \& White, S. 2000, ApJ, 530, 547

Jenkins, A., Frenk, C. S., Pearce, F. R., et al. 1998, ApJ, 499, 20

Kaiser, N. 1992, ApJ, 388, 272

Kaiser, N., Squires, G., Fahlman, G., \& Woods, D. 1994, in Clusters of Galaxies, ed. F. Durret, A. Mazure, \& J. Tran Thanh Van (Éditions Frontières), 437, 56

Kaiser, N., Squires, G., \& Broadhurst, T. 1995, ApJ, 449, 460
Kaiser, N., Wilson, G., Luppino, G. 2000 [astro-ph/0003338]

Lahav, O., Bridle, S. L., Percival, W. J., et al. 2001, submitted to MNRAS [astro-ph/0112162]

Maoli, R., Van Waerbeke, L., Mellier, Y., et al. 2001, A\&A, 368, 766

Massey, R., Bacon, D., Refregier, A., \& Ellis, R. 2001, in 2 pages. To appear in A New Era in Cosmology, ASP Conf. Proc., ed. T. Shanks, \& N. Metcalfe, 12393

Miralda-Escude, J. 1991, ApJ, 380, 1

Peacock, J. A., \& Dodds, S. J. 1996, MNRAS, 280, L19

Pen, U. 1998, ApJ, 498, 60

Pen, U., Van Waerbeke, L., \& Mellier, Y. 2002, ApJ, 567, 31

Pierpaoli, E., Scott, D., \& White, M. 2001, MNRAS, 325, 77

Rhodes, J., Refregier, A., \& Groth, E. J. 2001, ApJ, 552, L85

Schneider, P., van Waerbeke, L., Jain, B., \& Kruse, G. 1998, MNRAS, 296, 873

Schneider, P., van Waerbeke, L., \& Mellier, Y. 2002, A\&A, 389, 729

Scoccimarro, R., Zaldarriaga, M., \& Hui, L. 1999, ApJ, 527, 1

Seljak, U. 2001, Submitted to MNRAS [astro-ph/0111362]

Szalay, A., Jain, B., Matsubara, T., \& SDSS, c. 2002, The SDSS collaboration, submitted to ApJ [astro-ph/0107419]

van Waerbeke, L., Bernardeau, F., \& Mellier, Y. 1999, A\&A, 342, 15

Van Waerbeke, L., Mellier, Y., Erben, T., et al. 2000, A\&A, 358, 30

Van Waerbeke, L., Hamana, T., Scoccimarro, R., Colombi, S., \& Bernardeau, F. 2001a, MNRAS, 322, 918

Van Waerbeke, L., Mellier, Y., Radovich, M., et al. 2001b, A\&A, 374, 757

Viana, P. T. P., Nichol, R. C., \& Liddle, A. R. 2001, submitted to ApJL [astro-ph/0111394]

Wittman, D. M., Tyson, J. A., Kirkman, D., Dell'Antonio, I., \& Bernstein, G. 2000, Nature, 405, 143 